ESAIM: M2AN 49 (2015) 601-619

DOI: $10.1051 / \mathrm{m} 2 \mathrm{an} / 2014048$
ESAIM: Mathematical Modelling and Numerical Analysis

www.esaim-m2an.org

\title{
THE DISPERSIVE WAVE DYNAMICS OF A TWO-PHASE FLOW RELAXATION MODEL
}

\author{
Susanne Solem ${ }^{1}$, Peder Aursand $^{1}$ and Tore Flåtten ${ }^{2}$
}

\begin{abstract}
We consider a general Euler-type two-phase flow model with relaxation towards phase equilibrium. We provide a complete description of the transition between the wave dynamics of the homogeneous relaxation system and that of the local equilibrium approximation. In particular, we present generally valid analytical expressions for the amplifications and velocities of each Fourier component. This transitional wave dynamics is fully determined by only two dimensionless parameters; a stiffness parameter and the ratio of the sound velocities in the stiff and non-stiff limits. A direct calculation verifies that the stability criterion is precisely the subcharacteristic condition. We further prove a maximum principle in the transitional regime, similar in spirit to the subcharacteristic condition; the transitional wave speeds can never exceed the largest wave speed of the homogeneous relaxation system. Finally, we identify the existence of a critical region of wave numbers where the sonic waves completely disappear from the system. This region corresponds to the casus irreducibilis of the describing cubic polynomial.
\end{abstract}

Mathematics Subject Classification. 35L65, 15A18, 76T10.

Received October 25, 2013. Revised May 7, 2014

Published online 17 March 2015.

\section{INTRODUCTION}

Based on the classical approach of Baer-Nunziato [5], a common way of modeling non-equilibrium two-phase flows is through hyperbolic relaxation models $[2,3,7,12,21,25,26]$. Recently, there has been significant interest in models for cavitation where a metastable gas-liquid mixture is moving with a single velocity $[24,27,30]$.

In this paper, we consider 1D models of this type where thermal and mechanical equilibrium are assumed. Such simplified models have applications to two-phase pipeline flow relevant for environmental engineering and

Keywords and phrases. Relaxation, subcharacteristic condition, phase transfer.

1 Department of Mathematical Sciences, Norwegian University of Science and Technology (NTNU), NO-7491 Trondheim,

Norway. susanne.solem@ntnu.no; peder.aursand@math.ntnu.no

2 SINTEF Materials and Chemistry, P. O. Box 4760 Sluppen, NO-7465 Trondheim, Norway. Tore.Flatten@sintef.no 
the petroleum industry [18]. In particular, we look at models that can be written in the general form $[9,11,17]$

$$
\begin{aligned}
\partial_{t}\left(\alpha_{\mathrm{g}} \rho_{\mathrm{g}}\right)+\partial_{x}\left(\alpha_{\mathrm{g}} \rho_{\mathrm{g}} u\right) & =\frac{1}{\varepsilon}\left(\mu_{\ell}-\mu_{\mathrm{g}}\right), \\
\partial_{t}\left(\alpha_{\ell} \rho_{\ell}\right)+\partial_{x}\left(\alpha_{\ell} \rho_{\ell} u\right) & =-\frac{1}{\varepsilon}\left(\mu_{\ell}-\mu_{\mathrm{g}}\right), \\
\partial_{t}(\rho u)+\partial_{x}\left(\rho u^{2}+p\right) & =0 \\
\partial_{t} E+\partial_{x}(u(E+p)) & =0 .
\end{aligned}
$$

Herein, $\alpha_{i}$ is the volume fraction of phase $i$, satisfying

$$
\alpha_{\mathrm{g}}+\alpha_{\ell}=1
$$

Furthermore, $\rho_{i}$ is the density, and $u$ is the common velocity. We assume that we are in the metastable region, so that each phase is equipped with separate state relations

$$
\begin{aligned}
& p_{i}=p_{i}\left(\rho_{i}, e_{i}\right), \\
& T_{i}=T_{i}\left(\rho_{i}, e_{i}\right),
\end{aligned}
$$

where $e_{i}$ is the specific internal energy. The model is assumed to be in thermal and mechanical equilibrium, so that

$$
\begin{gathered}
T=T_{\mathrm{g}}=T_{\ell}, \\
p=p_{\mathrm{g}}=p_{\ell} .
\end{gathered}
$$

The total energy $E$ of the mixture is given by

$$
E=\epsilon+\frac{1}{2} \rho u^{2},
$$

where

$$
\begin{aligned}
& \rho=\rho_{\mathrm{g}} \alpha_{\mathrm{g}}+\rho_{\ell} \alpha_{\ell}, \\
& \epsilon=\rho_{\mathrm{g}} \alpha_{\mathrm{g}} e_{\mathrm{g}}+\rho_{\ell} \alpha_{\ell} e_{\ell}
\end{aligned}
$$

are the mixture density and internal energy, respectively. Finally, $\mu_{i}$ is the chemical potential given by

$$
\mu_{i}=e_{i}+\frac{p}{\rho_{i}}-T s_{i},
$$

where $s_{i}$ is the specific entropy of phase $i$.

In this paper, our focus is on purely mathematical analysis of this model. In particular, we aim to provide a complete as possible description of how the wave dynamics depend on the thermodynamic parameters and the strength of the relaxation term. Herein, our analysis remains generally valid for any state relations (1.3) and (1.4) satisfying standard thermodynamic stability conditions. We refer to [14] for a rigorous discussion on how the concepts of phase equilibrium and metastable states can be extended to simplified thermodynamic models like the stiffened-gas $[10,13,24]$ law.

For general hyperbolic relaxation systems, the zero relaxation limit has been the topic of much study $[16,22,23]$. In the system (1.1a)-(1.1d), $\varepsilon$ can be seen as a time-scale of the relaxation process. In the limit $\varepsilon \rightarrow \infty$, the phase compositions of the mixture are frozen and the model becomes a hyperbolic conservation law 
for the masses, the total momentum and the total energy. In the limit $\varepsilon \rightarrow 0$, the relaxation system is formally equivalent to the equilibrium system

$$
\begin{aligned}
\mu_{\mathrm{g}} & =\mu_{\ell}, \\
\partial_{t} \rho+\partial_{x}(\rho u) & =0, \\
\partial_{t}(\rho u)+\partial_{x}\left(\rho u^{2}+p\right) & =0 \\
\partial_{t} E+\partial_{x}(u(E+p)) & =0,
\end{aligned}
$$

where the two mass balance equations are replaced with a single conservation law for the total mass.

The wave dynamics of the limiting cases for $\varepsilon$ is well understood. In their recent work, Flåtten and Lund $[11,17]$ analyzed how the characteristic velocities of two-phase relaxation models depend on different assumptions on chemical, thermal and mechanical equilibrium. They found that for models of the type (1.1a)-(1.1d), the wave velocities of the equilibrium model will interlace those of the full model. This is known from the general theory of hyperbolic relaxation systems as the subcharacteristic condition, which may be derived from entropy considerations [8] and is closely related to the stability of the relaxation process [29].

However, in a practical application the relaxation parameter $\varepsilon$ will take finite values depending on the rate of mass transfer modeled by e.g. statistical rate theory [28]. Since the speed of sound in a pipeline can play a crucial role in important transient events such as crack propagation and emergency depressurization, this warrants the study of the wave dynamics in the transitional regime for which $\varepsilon$ takes finite values.

While hyperbolic relaxation systems have been an active field of research for decades, the development of a general theory for the wave-dynamics in this transitional regime has been limited. Recently, some of the present authors studied the transitional regime of general $2 \times 2$ relaxation systems [4]. In this case, the transitional wave speeds were found to fulfill a transitional subcharacteristic condition. Moreover, a critical transition point was found for which the wave dynamics abruptly change from being similar to the equilibrium system to behaving more like the frozen system.

The purpose of the present paper is to analyze the transitional wave dynamics of the $1 \mathrm{D}$ phase relaxation model (1.1a)-(1.1d), by studying the individual Fourier components following the approach of [4,29]. In particular, we will show how the wave dynamics are fully determined by only two dimensionless parameters; a stiffness parameter and the ratio of the sound velocities in the limiting equilibrium and frozen models.

Similarly to the observation made in [4], a critical transition region between the equilibrium dynamics and the frozen dynamics will be identified. In this region, the sonic waves lose their physical meaning and are replaced with an indeterminate wave moving with the fluid velocity. Mathematically, this interesting phenomenon corresponds exactly to the casus irreducibilis of the cubic polynomial whose roots describe the Fourier components.

This paper is organized as follows. In Section 2, we review the theory of plane wave solutions to linear relaxation systems. In Section 3, we present an explicit linearization of the two-phase flow relaxation system considered in this paper. In Section 4, we present the characteristic polynomial whose roots describe the velocities and amplifications of the transitional waves. This polynomial satisfies Galilean symmetry under a change of inertial reference frame, which greatly simplifies the calculations.

These roots are analyzed in Section 5. In Sections 5.1-5.2, we verify that we recover the frozen and equilibrium waves in the stiff and non-stiff limits. In Section 5.3, we verify that the transitional waves are stable if and only if the subcharacteristic condition is satisfied. In Section 5.4, we prove a principle that carries the essence of the subcharacteristic condition over to the transitional regime of finite relaxation time; for all wave numbers, the maximum wave speed of the relaxation system can never exceed the maximum wave speed of the frozen (non-stiff) limit of the system. In Section 5.5, we provide fully general closed-form expressions for the velocities and amplifications of the transitional waves.

In Section 6, we provide some interpretations and illustrations of these analytical results. In particular, we identify a critical region of wave numbers which appears if the ratio between the sound velocities in the stiff and non-stiff limits is sufficiently small. Through this critical region, corresponding to the casus irreducibilis of the describing cubic polynomial, all waves propagate with the fluid velocity $u$ and a continuous labeling of the 
separate waves becomes impossible. Hence this region can be interpreted as a set of wave numbers where the relaxation system, in a very qualitative way, changes character from behaving more like the equilibrium system to acting more like the frozen system.

Finally, in Section 7, the main insights of the paper are summarized.

\section{LINEARIZED RELAXATION SYSTEMS}

The transitional dynamics can be studied through linear analysis. A hyperbolic relaxation system such as the phase relaxation model (1.1a)-(1.1d) can be written in the general form [8]

$$
\partial_{t} \boldsymbol{U}+\partial_{x} \boldsymbol{F}(\boldsymbol{U})=\frac{1}{\varepsilon} \boldsymbol{Q}(\boldsymbol{U}),
$$

where $\boldsymbol{U}=\boldsymbol{U}(x, t) \in G \subseteq \mathbb{R}^{N}$ for some state space $G$.

Now let $\hat{\boldsymbol{U}}$ be some constant equilibrium state, characterized by

$$
\boldsymbol{Q}(\hat{\boldsymbol{U}})=0 .
$$

The relaxation system (2.1) linearized around $\hat{\boldsymbol{U}}$ can then be written as

$$
\partial_{t} \boldsymbol{V}+\boldsymbol{A} \partial_{x} \boldsymbol{V}=\frac{1}{\varepsilon} \boldsymbol{R} \boldsymbol{V}
$$

where

$$
\boldsymbol{V}=\boldsymbol{U}-\hat{\boldsymbol{U}}
$$

and the constant matrices

$$
\boldsymbol{A}=\frac{\partial \boldsymbol{F}}{\partial \boldsymbol{U}} \quad \text { and } \quad \boldsymbol{R}=\frac{\partial \boldsymbol{Q}}{\partial \boldsymbol{U}}
$$

are evaluated at the equilibrium state $\hat{\boldsymbol{U}}$.

Remark 2.1. Note that this linearization is valid also in the more general case where $\varepsilon=\varepsilon(\boldsymbol{U})$, if it can be assumed that $\varepsilon$ is differentiable at equilibrium. Define

$$
\hat{\varepsilon}=\varepsilon(\hat{\boldsymbol{U}}) .
$$

Then it follows from (2.2) that

$$
\frac{1}{\hat{\epsilon}} \boldsymbol{R}=\frac{\partial}{\partial \boldsymbol{U}}\left(\frac{1}{\varepsilon} \boldsymbol{Q}(\boldsymbol{U})\right)=\boldsymbol{Q}(\hat{\boldsymbol{U}}) \frac{\partial}{\partial \boldsymbol{U}}\left(\frac{1}{\varepsilon}\right)+\frac{1}{\hat{\varepsilon}} \frac{\partial \boldsymbol{Q}}{\partial \boldsymbol{U}}=\frac{1}{\hat{\varepsilon}} \frac{\partial \boldsymbol{Q}}{\partial \boldsymbol{U}} .
$$

\subsection{Plane-wave solutions}

For an initial condition $\boldsymbol{V}(x, 0) \in L^{2}(\mathbb{R})$, there exists a unique solution to $(2.3)$ [15]. If $\boldsymbol{V}(x, 0) \in L^{2}(\mathbb{T})$ with $\mathbb{T} \subset \mathbb{R}$ and $\mathbb{T}$ is of length $T<\infty$, the solution can be written in the general form

$$
\boldsymbol{V}(x, t)=\sum_{k} \boldsymbol{V}_{k}(x, t)=\sum_{k} \exp (\boldsymbol{H}(k) t) \exp (i k x) \hat{\boldsymbol{V}}(k),
$$

where $k$ is the wave number and $\boldsymbol{H}$ is a wave-number dependent matrix given by

$$
\boldsymbol{H}(k)=\frac{1}{\varepsilon} \boldsymbol{R}-i k \boldsymbol{A} .
$$

We now assume that $\boldsymbol{H}$ is diagonalizable, i.e. it can be written in the form

$$
\boldsymbol{H}(k)=\boldsymbol{P} \boldsymbol{\Lambda} \boldsymbol{P}^{-1},
$$


where $\boldsymbol{P}$ is its matrix of eigenvectors and $\boldsymbol{\Lambda}$ the diagonal matrix of eigenvalues. By using (2.10), we may then write the general solution (2.8) in terms of plane waves as

$$
\boldsymbol{V}(x, t)=\sum_{k} \sum_{j=1}^{N} \overline{\boldsymbol{V}}_{j}(k) \exp \left(i k x+\lambda_{j} t\right),
$$

for some amplitudes $\overline{\boldsymbol{V}}_{j}(k)$. Now to each eigenvalue $\lambda_{j}$ of $\boldsymbol{H}(k)$ there is an associated plane wave with velocity

$$
v_{j}(k)=-\frac{1}{k} \operatorname{Im}\left(\lambda_{j}\right),
$$

and amplification factor

$$
f_{j}(k)=\operatorname{Re}\left(\lambda_{j}\right)
$$

as can be seen by writing $(2.11)$ as

$$
\boldsymbol{V}(x, t)=\sum_{k} \sum_{j=1}^{N} \overline{\boldsymbol{V}}_{j}(k) \exp \left(f_{j} t\right) \exp \left(i k\left(x-v_{j} t\right)\right) .
$$

It now follows from (2.9) that $\boldsymbol{H}$ satisfies the symmetry

$$
\boldsymbol{H}(k)=\overline{\boldsymbol{H}(-k)} .
$$

Hence

$$
\lambda_{j}(k)=\overline{\lambda_{j}(-k)},
$$

and consequently

$$
\begin{aligned}
& f_{j}(k)=f_{j}(-k), \\
& v_{j}(k)=v_{j}(-k) .
\end{aligned}
$$

Hence we may with no loss of generality restrict the analysis of this paper to non-negative wave numbers, i.e. we assume

$$
k \in[0, \infty) .
$$

\section{The PhASE RELAXATION SYSTEM}

We now aim to derive an explicit expression for the matrix $\boldsymbol{H}$ corresponding to the phase relaxation system (1.1a)-(1.1d). In the context of (2.1), we have

$$
\boldsymbol{U}=\left[\begin{array}{c}
\rho_{\mathrm{g}} \alpha_{\mathrm{g}} \\
\rho_{\ell} \alpha_{\ell} \\
\rho u \\
E
\end{array}\right]
$$

From (1.3) and (1.4) we may derive the thermodynamic parameters [12]:

$$
\begin{gathered}
C_{p, i}=\rho_{i} \alpha_{i} c_{p, i}=\rho_{i} \alpha_{i} T\left(\frac{\partial s_{i}}{\partial T}\right)_{p}, \\
\zeta_{i}=\left(\frac{\partial T}{\partial p}\right)_{s_{i}}=-\frac{1}{\rho_{i}^{2}}\left(\frac{\partial \rho_{i}}{\partial s_{i}}\right)_{p} .
\end{gathered}
$$


The Jacobian matrix $\boldsymbol{A}$ for the system may now be obtained as outlined by Abgrall [1]. As stated by Propositions $9-10$ in [12] and Propositions 1-2 in [19], we have

$$
\boldsymbol{A}=\left[\begin{array}{cccc}
(1-Y) u & -Y u & Y & 0 \\
-(1-Y) u & Y u & 1-Y & 0 \\
a_{\mathrm{g}}-u^{2} & a_{\ell}-u^{2} & 2 u-u P_{\epsilon} & P_{\epsilon} \\
u\left(a_{\mathrm{g}}-\frac{E+p}{\rho}\right) u\left(a_{\ell}-\frac{E+p}{\rho}\right) & \frac{E+p}{\rho}-u^{2} P_{\epsilon} u\left(P_{\epsilon}+1\right)
\end{array}\right],
$$

where

$$
\begin{gathered}
Y=\frac{\rho_{\mathrm{g}} \alpha_{\mathrm{g}}}{\rho} \\
\mathcal{P}_{\epsilon}=\frac{\rho \tilde{c}^{2}}{T} \frac{\zeta_{\mathrm{g}} C_{p, \mathrm{~g}}+\zeta_{\ell} C_{p, \ell}}{C_{p, \mathrm{~g}}+C_{p, \ell}}
\end{gathered}
$$

and

$$
a_{i}=\frac{\rho \tilde{c}^{2}}{\rho_{i}}+\left(\frac{1}{2} u^{2}-e_{i}-\frac{p}{\rho_{i}}\right) \mathcal{P}_{\epsilon}, \quad i \in\{\mathrm{g}, \ell\} .
$$

Furthermore, $\tilde{c}$ is the sound velocity corresponding to the limit $\varepsilon \rightarrow \infty$ in (1.1a)-(1.1d), and is given by [12]:

$$
\tilde{c}^{-2}=\frac{\alpha_{\mathrm{g}}}{\rho_{\mathrm{g}} c_{\mathrm{g}}^{2}}+\frac{\alpha_{\ell}}{\rho_{\ell} c_{\ell}^{2}}+\frac{C_{p, \mathrm{~g}} C_{p, \ell}\left(\zeta_{\mathrm{g}}-\zeta_{\ell}\right)^{2}}{T\left(C_{p, \mathrm{~g}}+C_{p, \ell}\right)},
$$

where

$$
c_{i}^{2}=\left(\frac{\partial p}{\partial \rho_{i}}\right)_{s_{i}}
$$

are the one-phase sound velocities.

\subsection{The relaxation matrix}

In this section, we derive the linearized relaxation matrix $\boldsymbol{R}$, i.e. the Jacobian of the vector

$$
\boldsymbol{Q}=\left[\begin{array}{c}
\mu_{\ell}-\mu_{\mathrm{g}} \\
\mu_{\mathrm{g}}-\mu_{\ell} \\
0 \\
0
\end{array}\right]
$$

evaluated at equilibrium. We start with establishing a useful differential.

Lemma 3.1. The differential for the chemical potential difference can be written as

$$
\mathrm{d}\left(\mu_{\mathrm{g}}-\mu_{\ell}\right)=\left(\frac{\rho \tilde{c}^{2} \beta}{\rho_{\mathrm{g}}}-h_{\mathrm{g}} \mathcal{M}_{\epsilon}\right) \mathrm{d}\left(\rho_{\mathrm{g}} \alpha_{\mathrm{g}}\right)+\left(\frac{\rho \tilde{c}^{2} \beta}{\rho_{\ell}}-h_{\ell} \mathcal{M}_{\epsilon}\right) \mathrm{d}\left(\rho_{\ell} \alpha_{\ell}\right)+\mathcal{M}_{\epsilon} \mathrm{d} \epsilon
$$

where

$$
\begin{aligned}
\Theta & =\frac{\zeta_{\mathrm{g}} C_{p, \mathrm{~g}}+\zeta_{\ell} C_{p, \ell}}{C_{p, \mathrm{~g}}+C_{p, \ell}}, \\
\beta & =-\frac{1}{\rho^{2}}\left(\frac{\partial \rho}{\partial Y}\right)_{p, s}=\frac{1}{\rho_{\mathrm{g}}}-\frac{1}{\rho_{\ell}}+\Theta\left(s_{\ell}-s_{\mathrm{g}}\right), \\
\mathcal{M}_{\epsilon} & =\mathcal{P}_{\epsilon} \beta+\frac{s_{\ell}-s_{\mathrm{g}}}{C_{p, \mathrm{~g}}+C_{p, \ell}}, \\
h_{k} & =e_{k}+\frac{p}{\rho_{k}}, \quad k \in\{\mathrm{g}, \ell\} .
\end{aligned}
$$


Proof. From the Legendre transform on the fundamental differential

$$
\mathrm{d} e_{k}=T \mathrm{~d} s_{k}+\frac{p}{\rho_{k}^{2}} \mathrm{~d} \rho_{k}
$$

we obtain

$$
\mathrm{d}\left(\mu_{\mathrm{g}}-\mu_{\ell}\right)=\left(\frac{1}{\rho_{\mathrm{g}}}-\frac{1}{\rho_{\ell}}\right) \mathrm{d} p+\left(s_{\ell}-s_{\mathrm{g}}\right) \mathrm{d} T .
$$

Furthermore, the following relation was derived as (110) in Flåtten et al. [12]:

$$
\frac{\mathrm{d}\left(\rho_{\mathrm{g}} \alpha_{\mathrm{g}}\right)}{\rho_{\mathrm{g}}}+\frac{\mathrm{d}\left(\rho_{\ell} \alpha_{\ell}\right)}{\rho_{\ell}}=\left(\frac{\alpha_{\mathrm{g}}}{\rho_{\mathrm{g}} c_{\mathrm{g}}^{2}}+\frac{\alpha_{\ell}}{\rho_{\ell} c_{\ell}^{2}}+\frac{\zeta_{\mathrm{g}}^{2} C_{p, \mathrm{~g}}+\zeta_{\ell}^{2} C_{p, \ell}}{T}\right) \mathrm{d} p-\frac{\zeta_{\mathrm{g}} C_{p, \mathrm{~g}}+\zeta_{\ell} C_{p, \ell}}{T} \mathrm{~d} T,
$$

and from (122), (159) and (160) in [12] we have:

$$
\mathrm{d} p=\left(\frac{\rho \tilde{c}^{2}}{\rho_{\mathrm{g}}}-\mathcal{P}_{\epsilon} h_{\mathrm{g}}\right) \mathrm{d}\left(\rho_{\mathrm{g}} \alpha_{\mathrm{g}}\right)+\left(\frac{\rho \tilde{c}^{2}}{\rho_{\ell}}-\mathcal{P}_{\epsilon} h_{\ell}\right) \mathrm{d}\left(\rho_{\ell} \alpha_{\ell}\right)+\mathcal{P}_{\epsilon} \mathrm{d} \epsilon .
$$

The result now follows from substituting (3.18) and (3.19) in (3.17).

This result gives us an explicit formulation of the relaxation matrix.

Proposition 3.2. In the context of (2.5), the relaxation matrix $\boldsymbol{R}$ corresponding to the system (1.1a)-(1.1d) is given by

$$
\boldsymbol{R}=\left[\begin{array}{cccc}
-R_{1} & -R_{2} & -R_{3} & -R_{4} \\
R_{1} & R_{2} & R_{3} & R_{4} \\
0 & 0 & 0 & 0 \\
0 & 0 & 0 & 0
\end{array}\right]
$$

where

$$
\begin{aligned}
& R_{1}=\frac{\rho \tilde{c}^{2} \beta}{\rho_{\mathrm{g}}}+\left(\frac{1}{2} u^{2}-h_{\mathrm{g}}\right) \mathcal{M}_{\epsilon}, \\
& R_{2}=\frac{\rho \tilde{c}^{2} \beta}{\rho_{\ell}}+\left(\frac{1}{2} u^{2}-h_{\ell}\right) \mathcal{M}_{\epsilon}, \\
& R_{3}=-u \mathcal{M}_{\epsilon}, \\
& R_{4}=\mathcal{M}_{\epsilon} .
\end{aligned}
$$

Proof. With

$$
\boldsymbol{U}=\left[\begin{array}{c}
U_{1} \\
U_{2} \\
U_{3} \\
U_{4}
\end{array}\right]=\left[\begin{array}{c}
\rho_{\mathrm{g}} \alpha_{\mathrm{g}} \\
\rho_{\ell} \alpha_{\ell} \\
\rho u \\
E
\end{array}\right]
$$

we have the relations

$$
\begin{aligned}
\mathrm{d}\left(\rho_{\mathrm{g}} \alpha_{\mathrm{g}}\right) & =\mathrm{d} U_{1}, \\
\mathrm{~d}\left(\rho_{\ell} \alpha_{\ell}\right) & =\mathrm{d} U_{2}, \\
\mathrm{~d} \epsilon & =\frac{1}{2} u^{2}\left(\mathrm{~d} U_{1}+\mathrm{d} U_{2}\right)-u \mathrm{~d} U_{3}+\mathrm{d} U_{4} .
\end{aligned}
$$

The result now follows from substituting (3.26)-(3.28) into (3.11). 


\section{THE CHARACTERISTIC POLYNOMIAL}

From Proposition 3.2 and (3.4), we may now directly construct the wave-number dependent matrix $\boldsymbol{H}$ as defined by (2.9). In equilibrium, the chemical potentials (1.10) are equal, so we may write

$$
s_{\mathrm{g}}-s_{\ell}=\frac{h_{\mathrm{g}}-h_{\ell}}{T} .
$$

Then a direct calculation of the characteristic polynomial of $\boldsymbol{H}(k)$ gives:

$$
\begin{aligned}
\lambda^{4}+\left(\frac{\gamma \tilde{c}^{2}}{\varepsilon \hat{c}^{2}}+4 i k u\right) \lambda^{3}+\left(k^{2} \tilde{c}^{2}-6 k^{2} u^{2}+3 i k u \frac{\gamma \tilde{c}^{2}}{\varepsilon \hat{c}}\right) \lambda^{2}+ & \left(-3 \frac{k^{2} u^{2}}{\varepsilon} \gamma \frac{\tilde{c}^{2}}{\hat{c}^{2}}+\frac{k^{2}}{\varepsilon} \tilde{c}^{2} \gamma-4 i k^{3} u^{3}+2 i k^{3} u \tilde{c}^{2}\right) \lambda \\
& +k^{4} u^{2}\left(u^{2}-\tilde{c}^{2}\right)-i \frac{k^{3} u^{3}}{\varepsilon} \frac{\tilde{c}^{2}}{\hat{c}^{2}} \gamma+i \frac{k^{3} u}{\varepsilon} \tilde{c}^{2} \gamma=0,
\end{aligned}
$$

where the parameter $\hat{c}$ corresponds to the mixture sound velocity of the equilibrium system (1.11a)-(1.11b). It is given by $[11,20,26]$

$$
\hat{c}^{-2}=\rho\left(\frac{\alpha_{\mathrm{g}}}{\rho_{\mathrm{g}} c_{\mathrm{g}}^{2}}+\frac{\alpha_{\ell}}{\rho_{\ell} c_{\ell}^{2}}+T\left(C_{p, \mathrm{~g}}\left(\frac{\zeta_{\mathrm{g}}}{T}+W\right)^{2}+C_{p, \ell}\left(\frac{\zeta_{\ell}}{T}+W\right)^{2}\right)\right),
$$

where

$$
W=\frac{1}{\rho_{\mathrm{g}} \rho_{\ell}} \frac{\rho_{\mathrm{g}}-\rho_{\ell}}{h_{\mathrm{g}}-h_{\ell}}
$$

We have also introduced the shorthand

$$
\gamma=\frac{\left(h_{\mathrm{g}}-h_{\ell}\right)^{2}}{T\left(C_{p, \mathrm{~g}}+C_{p, \ell}\right)}
$$

Note that the sound velocities satisfy the subcharacteristic condition $[8,16]$

$$
\hat{c} \leq \tilde{c}
$$

subject only to fundamental thermodynamic stability constraints [11].

\subsection{Galilean invariance}

We can demonstrate that the roots of the characteristic polynomial possess the expected Galilean symmetry, i.e. they are invariant under a change of inertial reference frame.

By introducing the dimensionless parameters

$$
\varphi=k \varepsilon \frac{\hat{c}^{2}}{\gamma \tilde{c}}, \quad y=\frac{\lambda}{k \tilde{c}}, \quad r=\frac{\hat{c}}{\tilde{c}},
$$

we may transform the polynomial (4.2) to

$$
\varphi\left(y+i \frac{u}{\tilde{c}}\right)^{2}\left(y+i\left(\frac{u}{\tilde{c}}+1\right)\right)\left(y+i\left(\frac{u}{\tilde{c}}-1\right)\right)+\left(y+i \frac{u}{\tilde{c}}\right)\left(y+i\left(\frac{u}{\tilde{c}}+r\right)\right)\left(y+i\left(\frac{u}{\tilde{c}}-r\right)\right)=0 .
$$

From (4.8) it is clear that the change of variables

$$
z=y+i \frac{u}{\tilde{c}}
$$

yields the polynomial

$$
\varphi z^{2}(z+i)(z-i)+z(z+i r)(z-i r)=0
$$

which is indeed independent of the velocity $u$. 
It is worth noting that the simple polynomial (4.10) now gives a complete description of the wave-number dependent velocities and amplifications for a general relaxation model in the form (1.1a)-(1.1d). Remarkably, this dynamics is uniquely determined only by the parameters $\varphi$ and $r$ as defined by (4.7). And, as stated by Remark 2.1, the model presented in Section 3 is sufficiently general to represent any such model where the relaxation term satisfies only two natural properties:

- The mass transfer term disappears in equilibrium where $\mu_{\mathrm{g}}=\mu_{\ell}$.

- The relaxation parameter $\varepsilon$ is differentiable across the equilibrium state.

In the following sections, we will study the transitional wave dynamics in full detail.

\section{TRANSITIONAL WAVE DYNAMiCS}

We first consider the wave dynamics in the limiting cases $\varepsilon \rightarrow \infty$ and $\varepsilon \rightarrow 0$ respectively, corresponding to the frozen limit of (1.1a)-(1.1d) and the equilibrium limit (1.11a)-(1.11b).

\subsection{The frozen limit}

In the limit of infinite relaxation time, corresponding to $\varphi \rightarrow \infty$, the polynomial (4.10) reduces to

$$
z^{2}(z+i)(z-i)=0
$$

with the following 4 roots:

- $z=0$ with multiplicity 2 , corresponding to two waves with velocity $u$ and zero amplification.

- $z= \pm i$, corresponding to two waves with velocities $u \pm \tilde{c}$ and zero amplification.

This is precisely the wave structure of the frozen model (1.1a)-(1.1d) [11,12]. Herein, the two waves of velocity $u$ physically represent entropy and mass fraction waves, whereas the waves of velocity $u \pm \tilde{c}$ are sonic waves. Note that from (4.7), there are three distinct ways this frozen limit can be physically realized:

- the limit of infinite wave number $k \rightarrow \infty$;

- the limit of infinite relaxation time $\varepsilon \rightarrow \infty$;

- the limit of equal phasic enthalpies giving $\gamma \rightarrow 0$ in (4.5).

\subsection{The stiff limit}

In the limit of zero relaxation time, corresponding to $\varphi \rightarrow 0$, the polynomial (4.10) reduces to

$$
z(z+i r)(z-i r)=0 .
$$

This has the following 3 roots:

- $z=0$, corresponding to a wave with velocity $u$ and zero amplification.

- $z= \pm i r$, corresponding to two waves with velocities $u \pm \hat{c}$ and zero amplification.

Hence we recover the wave structure of the equilibrium model (1.11a)-(1.11d) [11,26]. Herein, the wave of velocity $u$ physically represents an entropy wave, whereas the waves of velocity $u \pm \hat{c}$ are sonic waves. From (4.7), there are now four distinct ways this stiff limit can be physically realized:

- the limit of zero wave number $k \rightarrow 0$;

- the limit of zero relaxation time $\varepsilon \rightarrow 0$;

- the limit of zero temperature giving $\gamma \rightarrow \infty$ in (4.5);

- the limit of zero heat capacities giving $\gamma \rightarrow \infty$ in (4.5).

Note that the limits considered above concern the wave-dynamics of the model. The state of the system must still be sufficiently close to equilibrium for the linearization to be valid. 


\subsection{Transitional stability}

The results of $[8,29]$ establish a direct link between the subcharacteristic condition (4.6) and the stability of the transitional waves as described by (4.10). Chen et al. [8] introduce an entropy condition which implies both the subcharacteristic condition and linear stability. Bouchut [6] proposes a reduced stability condition, weaker than the entropy condition, which also ensures that the subcharacteristic condition is satisfied.

Yong [29] proves that the subcharacteristic condition is necessary for linear stability of relaxation systems with a rank 1 relaxation operator.

For our current model, we here provide a concrete calculation showing that linear stability is equivalent to the subcharacteristic condition. We first observe that for all $\varphi,(4.10)$ has a trivial root $z=0$ corresponding to the entropy wave. Hence this wave may be fully described as follows; it will propagate with velocity $u$, and no amplification, independent of the wave number and relaxation time.

Eliminating this trivial root from (4.10), we obtain

$$
\varphi z\left(z^{2}+1\right)+\left(z^{2}+r^{2}\right)=0 .
$$

For the plane wave solutions (2.8) to be stable, the amplification factors (2.13) must be non-positive. This is equivalent to the requirement that the roots $z_{i}$ of (5.3) satisfy $\operatorname{Re} z_{i} \leq 0$.

Proposition 5.1 (Linear Stability). Let $\varphi \in(0, \infty)$. The real part of the roots of the polynomial (5.3) are nonpositive if and only if the subcharacteristic condition (4.6) is satisfied, i.e.

$$
0 \leq r \leq 1
$$

Proof. This general result follows as a special case of the Routh-Hurwitz theorem. For the purpose of illustration, we also state below a simple direct proof for the case where two of the roots are complex conjugate. A direct proof for the remaining case of distinct real roots may be derived along the same lines.

The polynomial (5.3) can be written in the form

$$
\begin{aligned}
z^{3}+\frac{1}{\varphi} z^{2}+z+\frac{r^{2}}{\varphi} & =\left(z-z_{2}\right)\left(z-z_{1}\right)\left(z-z_{0}\right) \\
& =z^{3}-\left(z_{2}+z_{1}+z_{0}\right) z^{2}+\left(z_{2} z_{1}+z_{1} z_{0}+z_{0} z_{2}\right) z-z_{2} z_{1} z_{0}=0 .
\end{aligned}
$$

We can, without loss of generality, assume $z_{2}$ is real while $z_{1}$ and $z_{0}$ are conjugate roots $\left(z_{0} z_{1}=\left|z_{0}\right|^{2}\right)$. Assume first that the polynomial is stable, i.e. Re $z_{i} \leq 0$. We can then write

$$
\begin{aligned}
\frac{1}{\varphi} \cdot 1 & =-\left(z_{2}+z_{1}+z_{0}\right)\left(z_{2} z_{1}+z_{1} z_{0}+z_{0} z_{2}\right) \\
& =\frac{r^{2}}{\varphi}-z_{2}^{2}\left(z_{0}+z_{1}\right)-\left|z_{0}\right|^{2}\left(z_{0}+z_{1}\right)-z_{2}\left(z_{0}+z_{1}\right)^{2} \geq \frac{r^{2}}{\varphi} .
\end{aligned}
$$

by using (5.5).

For the converse statement, assume $0 \leq r \leq 1$. From the positivity of $\varphi$ we have

$$
\frac{r^{2}}{\varphi}=-z_{2} z_{1} z_{0}=-z_{2}\left|z_{0}\right|^{2} \geq 0
$$

which directly implies $z_{2}=\operatorname{Re} z_{2} \leq 0$. It remains to show that $z_{0}+z_{1} \leq 0$. Using the assumption and (5.5) we can write, after multiplying by -1 ,

$$
z_{0}+z_{1}+z_{2} \leq z_{2}\left|z_{0}\right|^{2} .
$$

Furthermore, we have

$$
1=z_{2} z_{1}+z_{1} z_{0}+z_{0} z_{2}=\left|z_{0}\right|^{2}+z_{2}\left(z_{0}+z_{1}\right),
$$

so we can then infer

$$
z_{0}+z_{1} \leq z_{2}\left(\left|z_{0}\right|^{2}-1\right)=-z_{2}^{2}\left(z_{0}+z_{1}\right),
$$

which completes the proof. 


\subsection{A maximum principle}

For general $2 \times 2$ relaxation systems, it was shown by Aursand and Flåtten [4] that the transitional wave velocities always satisfy a monotonicity principle; the velocities $v_{j}(k)$ are in this case monotonic functions of the stiffness parameter $\varphi$.

As will be demonstrated in Section 6, this monotonicity principle does not carry over to the relaxation model considered in this paper. However, a weaker constraint on the velocities may be derived.

Proposition 5.2 (Maximum Principle). Let $\varphi \in(0, \infty)$. Assume that the subcharacteristic condition is satisfied, i.e. $r^{2} \leq 1$ in the context of (5.3). Then the imaginary parts of the roots in (5.3) satisfy $|\operatorname{Im}(z)| \leq 1$.

Proof. Let $z=a+i b$, where $b=\operatorname{Im}(z)$ and $a=\operatorname{Re}(z)$. Then (5.3) is equal to

$$
\varphi\left(a^{3}-3 a b^{2}+a\right)+a^{2}-b^{2}+r^{2}+i\left[\varphi\left(3 a^{2} b-b^{3}+b\right)+2 a b\right]=0 .
$$

Both the real and the imaginary part of (5.11) have to be equal to zero, giving us, for the imaginary part,

$$
b^{2}-1=\frac{2 a}{\varphi}+3 a^{2} .
$$

It follows from Proposition 5.1 that $a \leq 0$ when $r^{2} \leq 1$. If $a=0$, then $b^{2}=1$. For the case $a<0$, let us assume that $|b|>1$. Then $b^{2}-1>0$ and, dividing (5.12) by $a$, we are left with the inequality

$$
a<-\frac{2}{3 \varphi}
$$

since $a<0$. Multiplying the imaginary part of (5.11) with $a / b$ and subtracting it from 3 times the real part, we get

$$
-8 \varphi a b^{2}+2 \varphi a+a^{2}-3 b^{2}+3 r^{2}=0 .
$$

By subtracting $1 / 3$ times (5.12) from (5.14), we then have

$$
a\left(-8 \varphi b^{2}+2 \varphi-\frac{2}{3 \varphi}\right)=\frac{8}{3} b^{2}-3 r^{2}+\frac{1}{3} .
$$

With $a$ satisfying (5.13), we get

$$
-\frac{2}{3 \varphi}\left(-8 \varphi b^{2}+2 \varphi-\frac{2}{3 \varphi}\right)<\frac{8}{3} b^{2}-3 r^{2}+\frac{1}{3} .
$$

which results in the inequality

$$
-r^{2}>\frac{8 b^{2}}{9}-\frac{5}{9}+\frac{4}{27 \varphi^{2}} .
$$

With $|b|>1$ the right hand side in (5.17) is positive, but the left hand side is negative, which is a contradiction. Thus $|b| \leq 1$.

Physically, this result may be interpreted as a causality principle; the relaxation terms cannot be used to increase the velocity of information propagation in a stable system. Note that for our particular two-phase flow relaxation model, Proposition 5.2 is equivalent to the statement

$$
u-\tilde{c} \leq v_{j}(k) \leq u+\tilde{c} \quad \forall j, k .
$$




\subsection{Analytical solutions}

Given that (5.3) is a cubic polynomial, it can be solved exactly. In this section, we provide explicit expressions for the roots and provide some interpretations of the results.

The discriminant of $(5.3)$ is

$$
\Delta=-\left(\frac{2}{3}\left(3 \varphi^{2}-1\right)\right)^{2}-\left(r^{2}-\frac{1}{9}\right)\left(27 \varphi^{2} r^{2}-15 \varphi^{2}+4\right) .
$$

It will now be convenient to introduce the auxiliary variables

$$
\begin{aligned}
& s=r^{2}-\frac{1}{9}, \\
& \omega=3 \varphi^{2}-1,
\end{aligned}
$$

enabling us to write (5.19) in the simple form

$$
\Delta=-\left(\frac{2}{3} \omega-3 s\right)^{2}-9 s^{2} \omega .
$$

Now the nature of the roots of (5.3) are determined by the sign of the discriminant as follows:

D1: $\Delta<0$ : the equation (5.3) has one real root and two complex conjugate roots.

D2: $\Delta \geq 0$ : the equation (5.3) has three real roots (casus irreducibilis).

Note that in the context of the transformation (4.9), a real root corresponds to a wave of velocity $u$. Hence the situation D2 (casus irreducibilis) corresponds to a critical region in wave numbers and relaxation times where the transitional sound velocities become zero. In fact, as will be described in the following, no continuous labeling of the waves as "sonic" or "mass fraction" can be made through this critical region.

\subsubsection{Critical region}

We now define the critical region $\mathcal{C}(r)$ as

$$
\mathcal{C}(r):=\{\varphi \in(0, \infty): \Delta(\varphi, r) \geq 0\} .
$$

We may then state the following proposition.

Proposition 5.3. The equation (5.3) has three real roots if and only if

$$
\varphi \in \mathcal{C}(r)
$$

where

Herein, $\varphi_{\mathrm{c}}^{ \pm}$are given by:

$$
\mathcal{C}(r)= \begin{cases}\varnothing & \text { if } r>\frac{1}{3} \\ {\left[\varphi_{\mathrm{c}}^{-}, \varphi_{\mathrm{c}}^{+}\right]} & \text {if } r \in[0,1 / 3]\end{cases}
$$

$$
\begin{aligned}
& \varphi_{\mathrm{c}}^{-}=\frac{1}{2 \sqrt{2}} \sqrt{9\left(2 r^{2}-3 r^{4}\right)+1-\sqrt{\left(r^{2}-1\right)\left(9 r^{2}-1\right)^{3}}}, \\
& \varphi_{\mathrm{c}}^{+}=\frac{1}{2 \sqrt{2}} \sqrt{9\left(2 r^{2}-3 r^{4}\right)+1+\sqrt{\left(r^{2}-1\right)\left(9 r^{2}-1\right)^{3}}} .
\end{aligned}
$$

Proof. The discriminant (5.22) changes sign when the parameter $\omega$ satisfies

$$
\omega_{\text {crit }}=s\left(\frac{9}{2}-\frac{81}{8} s \pm \frac{27}{8} \sqrt{9 s^{2}-8 s}\right),
$$

which corresponds to real-valued $\varphi$ only if $r^{2} \leq 1 / 9$. In this case, the transformation (5.21) gives (5.26)-(5.27), which are unconditionally positive. 
We are now in the position to state explicit formulae for the roots inside and outside of the critical region.

\subsubsection{One real and two complex solutions}

We now assume that $\Delta<0$, i.e. $\varphi \notin \mathcal{C}(r)$. We now define

$$
Q=\frac{1}{2}\left(8 \omega-108 s \varphi^{2}+12 \varphi \sqrt{-3 \Delta}\right)^{1 / 3} .
$$

Then the real solution may be written as

$$
z_{1}=\frac{1}{3 \varphi}\left(Q-\frac{\omega}{Q}-1\right)
$$

whereas the complex solutions are given by

$$
z_{2,3}=-\frac{1}{6 \varphi}\left(Q-\frac{\omega}{Q}+2 \pm \sqrt{3} i\left(Q+\frac{\omega}{Q}\right)\right) .
$$

\subsubsection{Casus irreducibilis}

We now consider the case $\Delta \geq 0$, i.e. $\varphi \in \mathcal{C}(r)$. We may put the polynomial (5.3) in the reduced form

$$
t^{3}+p t+q=0
$$

where

$$
\begin{aligned}
& t=z+\frac{1}{3 \varphi}, \\
& p=\frac{3 \varphi^{2}-1}{3 \varphi^{2}}=\frac{\omega}{3 \varphi^{2}}, \\
& q=\frac{2-9 \varphi^{2}+27 r^{2} \varphi^{2}}{27 \varphi^{3}}=\frac{s}{\varphi}-\frac{2 \omega}{27 \varphi^{3}} .
\end{aligned}
$$

Now the roots are given by $(k=0,1,2)$,

$$
t_{k}=\frac{2}{3 \varphi \zeta} \cos \left(\frac{1}{3} \arccos \left(\frac{27 s \varphi^{2} \zeta^{3}}{2}+\zeta\right)+\frac{\pi(2 k-1)}{3}\right)
$$

where

This yields

$$
\zeta=\frac{1}{\sqrt{-\omega}}
$$

$$
z_{k}=\frac{1}{3 \varphi}\left(\frac{2}{\zeta} \cos \left(\frac{1}{3} \arccos \left(\frac{27 s \varphi^{2} \zeta^{3}}{2}+\zeta\right)+\frac{\pi(2 k-1)}{3}\right)-1\right)
$$

\section{Illustrations AND Discussion}

The analytical expressions derived in the previous section are too intangible to provide much in terms of direct insights into the structure of the transitional waves. In this section, we will illuminate this structure through a graphical investigation of the expressions (5.30)-(5.31) and (5.38).

As the roots are continuous functions of the stiffness parameter $\varphi$, one would expect that we should be able to identify sonic waves $P^{ \pm}$and a mass fraction wave $Y$ that are continuously transformed between the frozen and stiff limits. In fact, this naïve assumption breaks down in the critical region; herein, the waves "mix" and no continuous labeling is possible. Similarly to the critical point found for the the general $2 \times 2$ system [4], we may interpret the critical region as a well-defined "transitional regime" where the system changes character from being equilibrium-like to behaving more like the frozen system. This interpretation will be made precise in the following. 


\subsection{Frozen-like and equilibrium-like waves}

For the purposes of the ensuing discussion, it will be convenient to introduce the following labeling of the waves, as given by the complex roots of equation (4.10).

- Frozen-like sonic waves $P_{\mathrm{f}}^{ \pm}$, which continuously transform into the frozen sonic waves as the limit $\varphi \rightarrow \infty$ is approached.

- Equilibrium-like sonic waves $P_{\mathrm{e}}^{ \pm}$, which continuously transform into the equilibrium sonic waves as the limit $\varphi \rightarrow 0$ is approached.

- A frozen-like mass fraction wave $Y_{\mathrm{f}}$, which continuously transforms into the frozen mass fraction wave as the limit $\varphi \rightarrow \infty$ is approached.

- An equilibrium-like mass fraction wave $Y_{\mathrm{e}}$, whose amplification factor tends to $-\infty$ as the limit $\varphi \rightarrow \infty$ is approached: i.e. the wave is fully damped and completely disappears from the system.

- An indeterminate wave $X$ which exists only in the critical region. It can neither be interpreted as a mass fraction nor a sonic wave, but instead serves to connect the equilibrium-like and frozen-like versions of these waves.

- An entropy wave $S$, corresponding to the trivial root $z=0$ in (4.10). The dispersive dynamics of this wave is independent of $\varphi$; the velocity of propagation is $u$ and the amplification factor is 0 for all values of $\varphi$.

We now present the corresponding analytical expressions for each of these waves in turn, disregarding the trivial entropy wave. We consider first the case $r>1 / 3$, where there is no critical region $\mathcal{C}(r)$.

\subsubsection{Smooth transitional dynamics}

Consider now the case $r>1 / 3$. In this case, the transition between the frozen and equilibrium limits is smooth, and the equilibrium-like and frozen-like waves are identical. We define the following labeling of the solutions (5.30)-(5.31):

- Mass fraction wave:

$$
\begin{aligned}
& \operatorname{Im}\left(Y_{\mathrm{f}}\right)=\operatorname{Im}\left(Y_{\mathrm{e}}\right)=0, \\
& \operatorname{Re}\left(Y_{\mathrm{f}}\right)=\operatorname{Re}\left(Y_{\mathrm{e}}\right)=\frac{1}{3 \varphi}\left(Q-\frac{\omega}{Q}-1\right) .
\end{aligned}
$$

- Sonic waves:

$$
\begin{aligned}
& \operatorname{Re}\left(P_{\mathrm{f}}^{ \pm}\right)=\operatorname{Re}\left(P_{\mathrm{e}}^{ \pm}\right)=-\frac{1}{6 \varphi}\left(Q-\frac{\omega}{Q}+2\right), \\
& \operatorname{Im}\left(P_{\mathrm{f}}^{ \pm}\right)=\operatorname{Im}\left(P_{\mathrm{e}}^{ \pm}\right)= \pm \frac{1}{2 \sqrt{3} \varphi}\left(Q+\frac{\omega}{Q}\right) .
\end{aligned}
$$

The amplifications and velocities of these waves are plotted in Figure 1, for different values of the parameter $r \in[1 / 3,1]$. Herein, the variables are plotted as functions of a rescaled stiffness parameter $\chi$, given by:

$$
\chi(\varphi)=\frac{\varphi}{\varphi+1},
$$

in order to limit the plotting domain to $[0,1)$.

For $r=1 / 3$, a critical phenomenon emerges; at the point

$$
\varphi_{\mathrm{c}}=\sqrt{\frac{1}{3}}
$$

all the roots coincide, and the eigenspace of $\boldsymbol{H}$ as given by (2.9) degenerates. Hence the assumption (2.10) leading to the plane wave solutions (2.11) breaks down at this point. The solutions corresponding to the sonic 

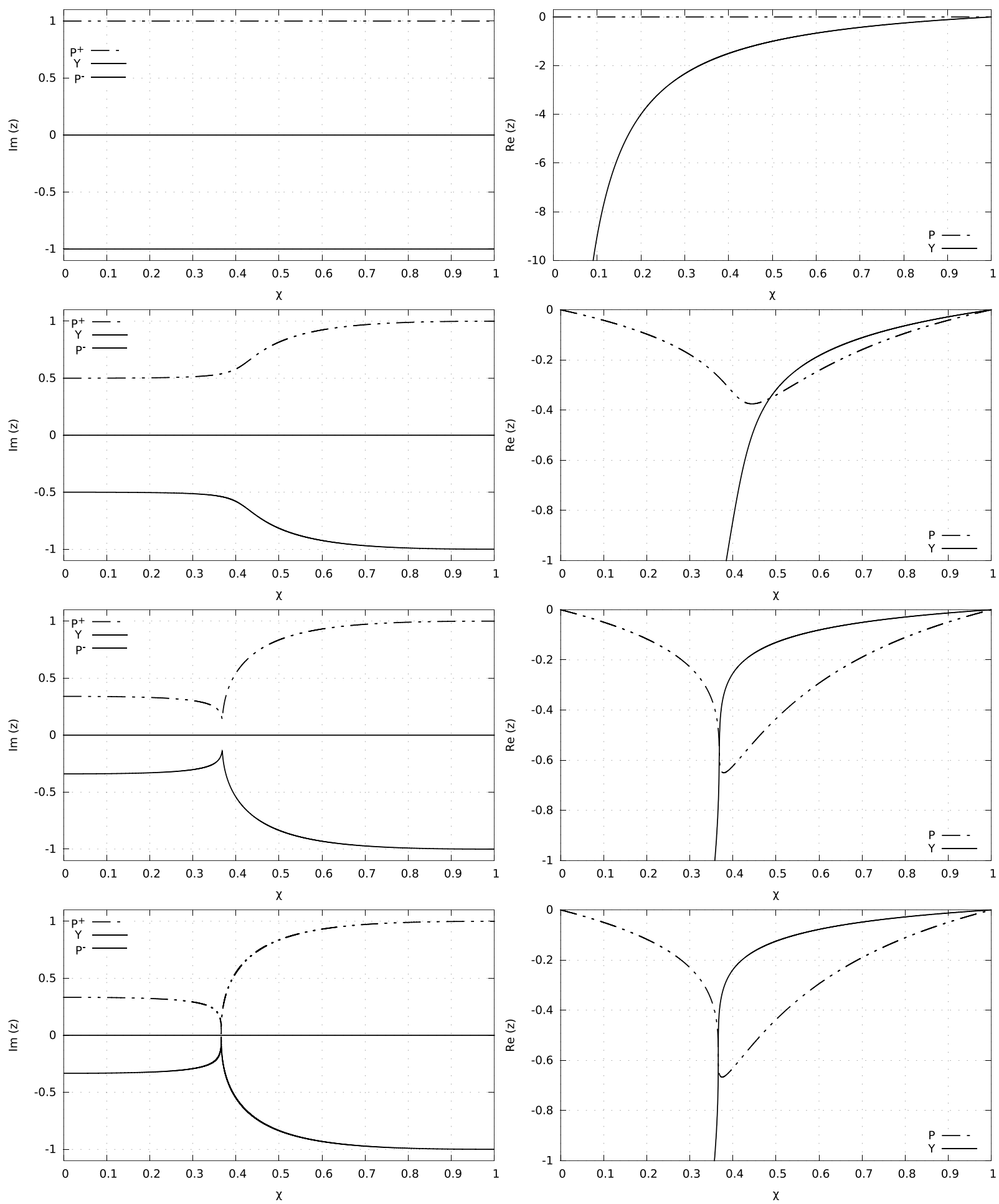

Figure 1. Transitional wave properties. $\operatorname{Left}: \operatorname{Im}(z)$ (velocities). Right: $\operatorname{Re}(z)$ (amplifications).

Top to bottom: $r=1.0, r=0.5, r=0.34, r=1 / 3$. 
velocities abruptly become zero, meaning that the sound waves in this limit propagate with the fluid velocity in the Eulerian frame. Also, around this point, the dampening of the sound waves is at its highest, and the dampening of the mass fraction wave (which disappears in the equilibrium system) increases sharply. Hence we can justify the statement that $\varphi_{\mathrm{c}}$ naturally divides the range of $\varphi$ into an equilibrium-like and frozen-like region.

Decreasing the parameter $r$ further causes this critical point $\varphi_{\mathrm{c}}$ to expand into a critical region. In this region, all the roots are distinct and the plane wave solutions (2.11) exist - but none of the solutions have a physical interpretation corresponding to sonic waves. This phenomenon will be investigated in the next section.

\subsubsection{Transition through critical region}

We consider now the case $r \in[0,1 / 3]$, i.e. there exists a critical region and we assume that the subcharacteristic condition $r \leq 1$ is satisfied (as indeed has been proved for our particular model [11]). We define the following labeling of the solutions (5.30)-(5.31) and (5.38):

- Mass fraction wave:

$$
\begin{gathered}
\operatorname{Im}\left(Y_{\mathrm{f}}\right)=0 \text { for } \varphi \geq \varphi_{\mathrm{c}}^{-}, \\
\operatorname{Im}\left(Y_{\mathrm{e}}\right)=0 \text { for } \varphi \leq \varphi_{\mathrm{c}}^{+},
\end{gathered}
$$

- Sonic waves:

$$
\begin{aligned}
& \operatorname{Re}\left(P_{\mathrm{f}}^{ \pm}\right)=-\frac{1}{6 \varphi}\left(Q-\frac{\omega}{Q}+2\right) \text { for } \varphi>\varphi_{\mathrm{c}}^{+}, \\
& \operatorname{Im}\left(P_{\mathrm{f}}^{ \pm}\right)= \pm \frac{1}{2 \sqrt{3} \varphi}\left(Q+\frac{\omega}{Q}\right) \text { for } \varphi>\varphi_{\mathrm{c}}^{+}, \\
& \operatorname{Re}\left(P_{\mathrm{e}}^{ \pm}\right)=-\frac{1}{6 \varphi}\left(Q-\frac{\omega}{Q}+2\right) \text { for } \varphi<\varphi_{\mathrm{c}}^{-}, \\
& \operatorname{Im}\left(P_{\mathrm{e}}^{ \pm}\right)= \pm \frac{1}{2 \sqrt{3} \varphi}\left(Q+\frac{\omega}{Q}\right) \text { for } \varphi<\varphi_{\mathrm{c}}^{-} .
\end{aligned}
$$

- Indeterminate wave:

$$
\begin{gathered}
\operatorname{Im}(X)=0 \text { for } \varphi \in\left[\varphi_{\mathrm{c}}^{-}, \varphi_{\mathrm{c}}^{+}\right] \\
\operatorname{Re}(X)=\frac{1}{3 \varphi}\left(\frac{2}{\zeta} \cos \left(\frac{1}{3} \arccos \left(\frac{27 s \varphi^{2} \zeta^{3}}{2}+\zeta\right)+\frac{\pi}{3}\right)-1\right) \quad \text { for } \varphi \in\left[\varphi_{\mathrm{c}}^{-}, \varphi_{\mathrm{c}}^{+}\right] .
\end{gathered}
$$

As we will see, this is the natural labeling if we want to assign a continuous physical interpretation of each wave between the branching points where the roots change character from being complex to being real.

The amplifications and velocities of these waves are plotted in Figure 2 as functions of $\chi$ as given by (6.5). Note in particular that the frozen-like and equilibrium-like mass fraction waves $Y_{\mathrm{f}}$ and $Y_{\mathrm{e}}$ overlap in the critical region; herein, they are truly separate waves. They are "backwardly connected" by the indeterminate wave $X$, which has no physical interpretation in terms of the waves existing in the frozen and equilibrium limit systems. Note that the wave $X$ also serves to connect the frozen-like and equilibrium-like sonic waves. 

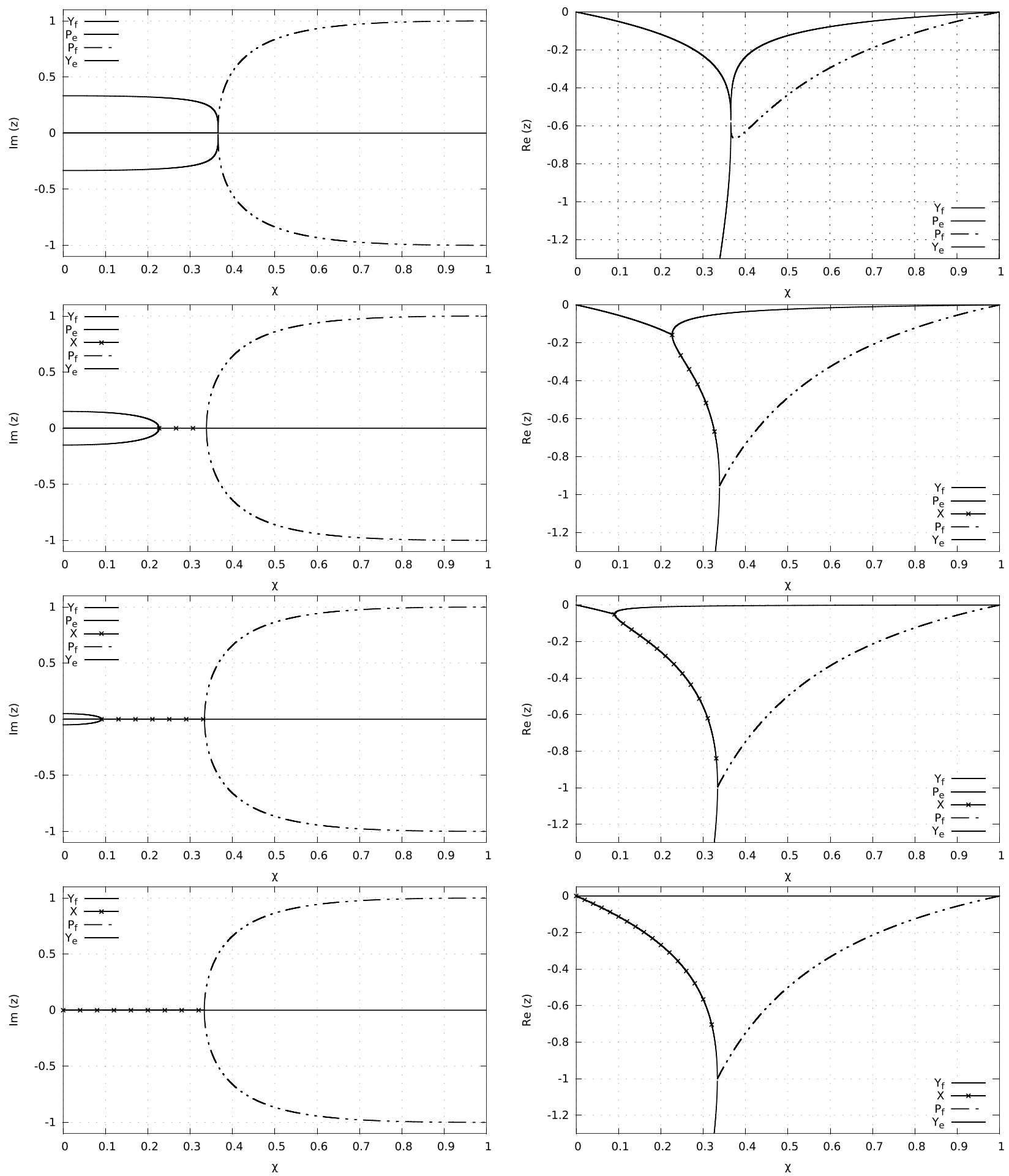

FiguRE 2. Transitional wave properties. Left: $\operatorname{Im}(z)$ (velocities). Right: $\operatorname{Re}(z)$ (amplifications). Top to bottom: $r=1 / 3, r=0.15, r=0.05, r=0$. 


\subsection{Directions for further work}

A highly relevant question is how the existence of the critical region will manifest itself in the solutions to the full nonlinear model, and how these solutions are related to the actual physics of two-phase flows with phase transfer. From the analysis, it is reasonable to expect that sound waves with wave numbers belonging to the critical region should not be able to propagate through the two-phase medium modelled by (1.1a)(1.1d). At present, we are not aware of any experiments that may be directly connected to this mathematical phenomenon. It would also be of interest to numerically investigate the behaviour of solutions to the nonlinear model (1.1a)-(1.1d) in the critical region.

It should be noted that the critical region considered here is certainly physically feasible; as demonstrated in [11], both water at 1 bar and $\mathrm{CO}_{2}$ at 50 bar satisfy $r<1 / 3$ at phase equilibrium for liquid volume fractions less than 0.05 .

We also note that the relaxation processes considered in $[11,17]$ all have the similar relationship between the eigenstructures of the frozen and stiff limits. Hence it is probable that similar critical phenomena may arise in these models.

Furthermore, the model (1.1a)-(1.1d) makes the assumption that the relaxation times for heat, volume and momentum transfer are negligible compared to phase transfer. Consequently, it would be of great interest to perform a similar analysis on two-phase flow models with several simultaneous relaxation processes.

\section{Summary}

We have investigated the wave dynamics of a two-phase flow model with relaxation towards phase equilibrium. We have shown that for any given thermodynamic substance, physical state, relaxation rate and chosen wave number $k$, the velocities and amplifications of the resulting plane waves are determined by only two dimensionless numbers (denoted as $r$ and $\varphi$ in our paper). This, combined with the Galilean symmetry of the model, allows for the highly simplified formulation (4.10) from which generally valid analytical results can be extracted. In particular, we obtain closed-form expressions for the wave-number dependent wave velocities and amplifications for any relaxation time. These analytical results unveil what is the main insight of this paper: if the ratio behind the stiff and non-stiff sound velocities is sufficiently small, a critical region in the wave numbers emerges, defining a non-smooth transition between the equilibrium and frozen limits. This extends the previous similar observation of Aursand and Flåtten [4] for general $2 \times 2$ systems.

Acknowledgements. The authors gratefully acknowledge partial financial support from the SINTEF Group. The third author was supported in part by the Research Council of Norway through the SIMCOFLOW project.

We are grateful to the anonymous reviewers who provided valuable comments to the first version of this paper.

\section{REFERENCES}

[1] R. Abgrall, An extension of Roe's upwind scheme to algebraic equilibrium real gas models. Comput. Fluids 19 (1991) 171-182.

[2] R. Abgrall and R. Saurel, Discrete equations for physical and numerical compressible multiphase mixtures. J. Comput. Phys. 186 (2003) 361-396.

[3] G. Allaire, S. Clerc and S. Kokh, A five-equation model for the simulation of interfaces between compressible fluids. J. Comput. Phys. 181 (2002) 577-616.

[4] P. Aursand and T. Flåtten, On the dispersive wave-dynamics of $2 \times 2$ relaxation systems. J. Hyperbolic Diff. Eq. 9 (2012) 641-659.

[5] M.R. Baer and J.W. Nunziato, A two-phase mixture theory for the deflagration-to-detonation transition (DDT) in reactive granular materials. Int. J. Multiphase Flow 12 (1986) 861-889.

[6] F. Bouchut, A reduced stability condition for nonlinear relaxation to conservation laws. J. Hyperbolic Diff. Eq. 1 (2004) 149-170.

[7] F. Caro, F. Coquel, D. Jamet and S. Kokh, A simple finite-volume method for compressible isothermal two-phase flows simulation. Int. J. Finite $\mathbf{3}$ (2006).

[8] G.-Q. Chen, C.D. Levermore and T.-P. Liu, Hyperbolic conservation laws with stiff relaxation terms and entropy. Commun. Pure Appl. Math. 47 (1994) 787-830. 
[9] S. Dellacherie, Relaxation schemes for the multicomponent Euler system. ESAIM: M2AN 37 (2003) 909-936.

[10] G. Faccanoni, S. Kokh and G. Allaire, Modelling and simulation of liquid-vapor phase transition in compressible flows based on thermodynamical equilibrium. ESAIM: M2AN 46 (2012) 1029-1054.

[11] T. Flåtten and H. Lund, Relaxation two-phase flow models and the subcharacteristic condition. Math. Models Methods Appl. Sci. 21 (2011) 2379-2407.

[12] T. Flåtten, A. Morin and S.T. Munkejord, Wave propagation in multicomponent flow models. SIAM J. Appl. Math. 70 (2010) $2861-2882$.

[13] T. Flåtten, A. Morin and S.T. Munkejord, On solutions to equilibrium problems for systems of stiffened gases. SIAM J. Appl. Math. 71 (2011) 41-67.

[14] P. Helluy and N. Seguin, Relaxation models of phase transition flows. ESAIM: M2AN 40 (2006) 331-352.

[15] H.-O. Kreiss and J. Lorenz, Initial-boundary value problems and the Navier-Stokes equations. Academic Press (1989).

[16] T.-P. Liu, Hyperbolic conservation laws with relaxation. Commun. Math. Phys. 108 (1987) 153-175.

[17] H. Lund, A hierarchy of relaxation models for two-phase flow. SIAM J. Appl. Math. 72 (2012) $1713-1741$.

[18] H. Lund and P. Aursand, Two-phase flow of $\mathrm{CO}_{2}$ with phase transfer. Energy Procedia 23 (2012) 246-255.

[19] A. Morin, P.K. Aursand, T. Flåtten and S.T. Munkejord, Numerical resolution of $\mathrm{CO}_{2}$ transport dynamics. In Proc. of SIAM Conference on Mathematics for Industry: Challenges and Frontiers (MI09). SIAM, Philadelphia (2009) 108-119.

[20] A. Morin and T. Flåtten, A two-fluid four-equation model with instantaneous thermodynamical equilibrium. Submitted.

[21] A. Murrone and H. Guillard, A five equation reduced model for compressible two phase flow problems. J. Comput. Phys. 202 (2005) 664-698.

[22] R. Natalini, Convergence to equilibrium for the relaxation approximation of conservation laws. Commun. Pure Appl. Math. 49 (1996) 795-823.

[23] R. Natalini, Recent results on hyperbolic relaxation problems. Analysis of systems of conservation laws. In vol. 99 of Chapman 83 Hall/CRC Monogr. Surv. Pure Appl. Math. Chapman \& Hall/CRC, Boca Raton, FL (1999) 128-198.

[24] M. Pelanti and K.-M. Shyue, A mixture-energy-consistent six-equation two-phase numerical model for fluids with interfaces, cavitation and evaporation waves. J. Comput. Phys. 259 (2014) 331-357.

[25] R. Saurel and R. Abgrall, A multiphase Godunov method for compressible multifluid and multiphase flows. J. Comput. Phys. 150 (1999) 425-467.

[26] R. Saurel, F. Petitpas and R. Abgrall, Modelling phase transition in metastable liquids: application to cavitating and flashing flows. J. Fluid Mech. 607 (2008) 313-350.

[27] R. Saurel, F. Petitpas and R.A. Berry, Simple and efficient relaxation methods for interfaces separating compressible fluids, cavitating flows and shocks in multiphase mixtures. J. Comput. Phys. 228 (2009) 1678-1712.

[28] C.A. Ward and G. Fang, Expression for predicting liquid evaporation flux: Statistical rate theory approach. Phys. Rev. E 59 (1999) 429-440.

[29] W.-A. Yong, Basic aspects of hyperbolic relaxation systems, in Advances in the Theory of Shock Waves. Vol. 47 of Progr. Nonlin. Differ. Eq. Appl. Birkhäuser Boston, Boston (2001) 259-305.

[30] A. Zein, M. Hantke and G. Warnecke, Modeling phase transition for compressible two-phase flows applied to metastable liquids. J. Comput. Phys. 229 (2010) 2964-2998. 